\title{
TU3A-(13)-5
}

\section{Design of Distributed and Discrete Broadband Amplifier Systems with Accurate Gain Profile and Optimal Fiber Combination}

\author{
Jer-Shien Chen, Hen-Wai Tsao, and *San-Liang Lee \\ Graduate Institute of Communication Engineering and Department of Electrical Engineering, \\ National Taiwan University, Taipei, Taiwan. \\ * Department of Electronic Engineering \\ National Taiwan University of Science and Technology, Taipei, Taiwan.
}

TEL : 886-2-27376401 Fax 886-2-27376424, E-mail: sanlee@et.ntust.edu.tw

Abstract----We point out the limitation of using an approximate gain profile in designing distributed fiber Raman amplifier (FRA) and design a broadband FRA based on the measured gain profile. A design of discrete amplifiers for ultra-long-haul transmission is also reported by optimizing the combination of DCF and SMF fibers.

Based on the approximate Raman gain profile, we designed a pumping scheme for a distributed FRA system to have flat bandwidth of $100 \mathrm{~nm}$ wide, as shown in Fig.1. However, when we replace the approximate Raman gain with the measured gain profile, the gain spectrum becomes quite different and the flatness disappears, as shown in Fig.1. In fact, the measured Raman gain curve degrades abruptly when the frequency shift is beyond the maximum Stoke shift, 13.2THz, but the approximate Raman gain curve doesn't. In designing a broadband FRA system, in order for considering the practical interactions among the pumps and signals, the simulated range of the Raman gain spectrum must be wide enough (e.g., at least $23.2 \mathrm{THz}$ for a 100 -channel system of $100 \mathrm{GHz}$ channel spacing). In such a case, the approximate gain profile results in a considerable error. To simplify the design procedure of a broadband FRA system, the simple method proposed here includes three steps: (1) Setting pumping frequency with equal interval, (2) Setting initial pumping power as a geometric series, and (3) adjusting the power of each pumping source. Then, flat gain is achieved easily, as shown in Fig. 2 .

In uitra-long-haul systems, we report a systematic design of a $100 \mathrm{~km}$-span long haul system without using FRAs by carefully inserting a segment of DCF into each span. We first evaluate different types of fiber combination for a single span. Thirty channels of $100 \mathrm{GHz}$ spacing in the $\mathrm{C}$-band are simulated and each carry a PRBS signal with a data rate of $10 \mathrm{Gbps}$. The configuration of $\left[\mathrm{SMF}_{1}+\mathrm{DCF}+\mathrm{SMF}_{2}\right]$ has the best performance. We then analyze the system performance for different output signal levels for a cascaded link. Under the condition of a BER less than $10^{-9}$, the maximal achievable transmission distances for the amplifier output power of $+7 \mathrm{dBm},+10 \mathrm{dBm}$, and $+13 \mathrm{dBm} "$, are about $300 \mathrm{~km}, 700 \mathrm{~km}$, and $1300 \mathrm{~km}$, respectively, as shown in Fig. 3 .

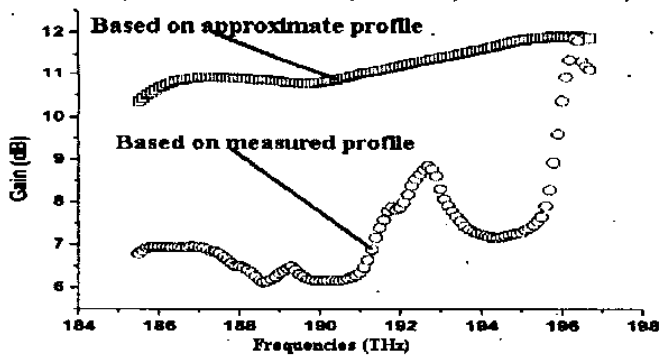

Fig. 1: Comparison of a 4-pump FRA gain with different gain profile.

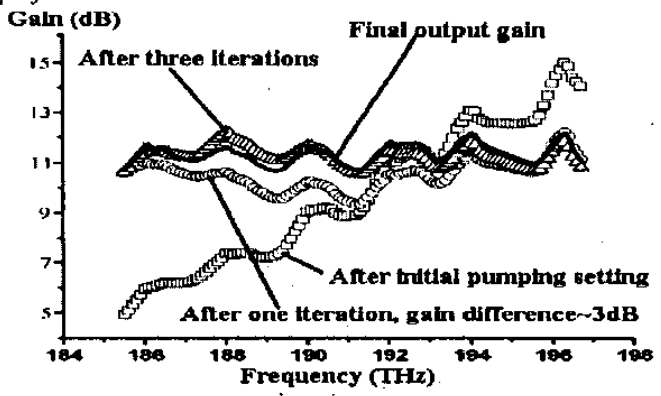

Fig. 2: Gain of the broadband FRA system.

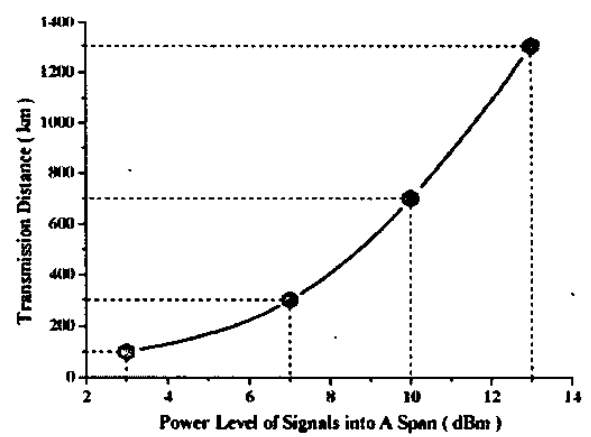

Fig.3: Maximal altainable distance against the output power. Reference:

[1]H. Kidorf et al., "Pump Interactions in a 100nm Bandwidth Raman Amplifier," IEEE Photon. Technol. Lett., vol.11, pp.530-532, 1999

[2]VPIcomponent Maker ${ }^{\text {TM }}$ Fiber Amplifier User's Manual, VPIsystems Inc., 2001

[3]R. Hainberger et al.,"Optimum span configuration of Raman-amplified dispersion-managed fibers," Proc. OFC '2001, pp. MI5-1-MIS-3 